\title{
Engajamento social da universidade: uma análise sobre a interação universidade- sociedade no Rio Grande do Sul
}

Naomi Moura Fujimoto (UFRGS);

Ana Lúcia Tatsch (UFRGS);

A partir da discussão recente sobre o engajamento social da universidade, este artigo objetiva compreender como se dão as interações universidade-sociedade no Rio Grande do Sul. Para tanto, utilizase como base de dados o Censo 2016 do Diretório dos Grupos de Pesquisa do Conselho Nacional de Desenvolvimento Científico e Tecnológico (DGP/CNPq). A análise visa identificar com quais agentes as universidades do Rio Grande do Sul interagem e que tipos de interação ocorrem, bem como as diferenças entre as áreas do conhecimento e entre as universidades públicas e privadas. Identificou-se que grupos de pesquisa de universidades do Rio Grande do Sul interagem com diversos agentes, como governo, associações, cooperativas, fundações, empresas e outras universidades, localizados especialmente no eixo sul-sudeste do Brasil.

palavras-chave:

Interações universidade-sociedade; Sistemas Nacionais de Inovação; Grupos de pesquisa.

Código JEL: I23, O30

Área Temática: 4.4 Redes de inovação. 


\section{Introdução}

Num contexto de complexificação do conhecimento científico e tecnológico, o papel das universidades de suporte às atividades inovativas empresariais tem sido alvo de vários estudos. Logo, a discussão sobre a interação universidade-firma ganhou espaço na literatura. Porém, mais do que nunca, é necessário estudar como as instituições de ensino superior se relacionam com outros atores. Em tempos de pandemia global, temos a oportunidade de refletir sobre o papel da pesquisa e da produção científica em prol de toda a população, atuando nas mais diversas esferas da sociedade e não apenas na produção de bens.

Segundo a literatura sobre Sistemas Nacionais de Inovação, as interações entre diversas instituições e atores presentes em um país contribuem para a geração de inovações e desenvolvimento socioeconômico. As universidades, particularmente, ocupam um papel central nas análises de diversos autores, sendo responsáveis pela geração e difusão de conhecimentos e tecnologia.

No caso dos países em desenvolvimento, as pesquisas em torno do tema não devem se basear nos mesmos critérios que as realizadas em países desenvolvidos. Neles existe uma necessidade maior de solucionar problemas sociais, tais como saneamento básico, desigualdade racial, desigualdade de gênero, acesso à educação e à saúde. Dessa maneira, torna-se importante analisar a interação da universidade com diversos agentes da sociedade e não apenas com a indústria. Esse engajamento envolve interações englobando também o governo, cooperativas, ONGs, hospitais, outras universidades, entre outros.

Diante disso, o objetivo geral desse artigo é investigar as interações universidade-sociedade no Rio Grande do Sul, identificando padrões de comportamento entre os grupos de pesquisa do estado e outros agentes da sociedade. Os objetivos específicos são analisar com quais agentes as universidades interagem, quais tipos de interação ocorrem e descobrir se existem diferenças entre as áreas de conhecimento e entre instituições de ensino superior públicas e privadas. Assim, pretende-se contribuir para o avanço da pesquisa sobre a interação universidade-sociedade no Brasil.

Para a realização desse estudo é feita uma análise de dados do censo do Diretório dos Grupos de Pesquisa do Conselho Nacional de Desenvolvimento Científico e Tecnológico (DGP/CNPq) de 2016. Essa base de dados é coletada bianualmente pelo diretório e reúne informações sobre os principais parceiros dos grupos de pesquisa brasileiros ligados a universidades, bem como os tipos de relações estabelecidas entre eles.

Esse artigo está organizado em cinco seções, incluindo essa introdução. Na segunda seção apresenta-se o referencial teórico, contextualizando a importância do engajamento social da universidade. Na terceira seção apresenta-se a metodologia de construção da base de dados. Na quarta seção, analisa-se os dados do censo do DGP/CNPq, relacionando-os à teoria revisada na sessão dois. Por último, as considerações finais sintetizam os principais resultados do trabalho e suas interpretações.

\section{O Engajamento Social da Universidade}

Na abordagem neo-schumpeteriana dos Sistemas Nacionais de Inovação (SNI) (FREEMAN, 1987; 1995; LUNDVALL, 1992), as instituições de ensino superior possuem um papel central. Para Lundvall (2007), as universidades são instituições líderes no SNI. Muito além de formar profissionais qualificados para trabalhar nas empresas, elas produzem conhecimento de base necessário para o desenvolvimento tecnológico. Já Nelson (1993) sublinha dois importantes papeis das universidades: o ensino para formar novos profissionais e a pesquisa na área tecnológica. A partir deste entendimento, as universidades têm sido foco de análise de vários estudos, especialmente daqueles que investigam as relações universidadeempresa (U-E).

Em países em desenvolvimento, no entanto, verifica-se que esse tipo de interação é pouco frequente. Para Arocena, Göransson e Sutz (2018), as universidades no subdesenvolvimento enfrentam dois obstáculos: a baixa proporção de jovens cursando ensino superior e a escassez de empregos em que os profissionais qualificados podem aplicar o conhecimento adquirido. Para o caso brasileiro, Suzigan e Albuquerque (2011) apontam três fatores adversos para a formação das relações universidade-empresa no 
Brasil: (i) o processo demorado de construção de institutos de pesquisa e instituições de ensino superior no país, (ii) a industrialização tardia e (iii) o atraso na criação de instituições monetárias e financeiras nacionais.

Diante deste contexto, estudos mais recentes têm procurado examinar as interações das universidades para além daquelas com empresas. Interações essas que ressaltam o engajamento da universidade com a sociedade, focada em atender os problemas sociais da região ou do país. Ainda para Arocena, Göransson e Sutz (2018), as universidades quando atuam de maneira a cooperar com a redução da desigualdade e do subdesenvolvimento, passam a ser vistas como "developmental universities".

Nesta direção, Kruss et al. (2012) estudam o engajamento social de instituições de ensino superior na África do Sul. Nesse país, assim como no Brasil, existem altas taxas de desemprego e desigualdade social, portanto os autores acreditam que iniciativas de desenvolvimento comunitário deveriam ser consideradas atividades relevantes para a universidade, afinal é impossível ignorar questões sociais em países pobres quando se fala no papel da universidade na geração de desenvolvimento.

It has been argued that national innovation research in such contexts should focus not only on capability building for the technological upgrading of firms, but equally, [...] mobilizing science, technology, and innovation to address problems of health, environmental sustainability, food security and disease" (KRUSS et al., 2012, p. 2).

Os autores realizaram um estudo empírico com o objetivo de mapear as formas e a escala das interações de universidades sul-africanas com parceiros externos por área do conhecimento e tipo de universidade, e identificar como as políticas e mecanismos de cada uma resultam em padrões diferentes de interação. Foram selecionadas cinco universidades: duas universidades de pesquisa, uma universidade rural, uma universidade de tecnologia e uma universidade multidisciplinar. Os dados foram coletados através de uma pesquisa realizada com uma amostra representativa de acadêmicos, relacionando-os às suas respectivas instituições.

Como resultado, foi encontrado que $81 \%$ dos acadêmicos entrevistados consideram que interagem com parceiros externos. Porém, uma conclusão interessante é que as cinco universidades incluídas no estudo possuem padrões significativamente diferentes: a universidade rural apresentou maior probabilidade de engajamento com o ensino, enquanto a universidade de tecnologia apresentou maior tendência de engajamento com firmas e o governo (KRUSS et al, 2012, p. 53).

Em países desenvolvidos a análise sobre o engajamento social da universidade também desperta curiosidade. No Reino Unido, frente à recessão provocada pela crise de 2008 e preocupado com o aquecimento global e o envelhecimento da população, Goddard (2009) defende que as universidades públicas devem cumprir seu dever civil (civic duty) engajando-se com parceiros externos nas esferas regional, nacional e global para promover o bem-estar econômico e social. Ele defende que o engajamento social deve ser além de uma "terceira missão", que teria menos recursos que o ensino e a pesquisa. Em vez disso, o engajamento social deve se tornar um princípio intrínseco da organização.

The civic university has a key role to play in fostering such system wide innovation and tackling the big challenges that confront the modern world, for example the need for sustainable cities or the many challenges and opportunities that arise from an ageing population. It can do this by serving public as well as private interests and embracing business and the community found outside its front door, connecting these communities to the global arena (GODDARD, 2009, p. 10).

Goddard (2009) escreve sobre o exemplo da Universidade Newcastle, no Reino Unido, para ilustrar como uma instituição de ensino superior poderia atuar no país de maneira socialmente engajada. Essa universidade esteve distanciada da sua comunidade desde a sua fundação, até que nos anos 1990, após uma nova fonte de financiamento voltada para o apoio do comércio local e a criação da Agência de Desenvolvimento Regional, a instituição passou envolver-se com a administração da cidade, proporcionando estudos na área de fertilidade, inovação para pequenas e médias empresas, abertura de teatros e realização de programas culturais. A cidade foi, então, designada como uma 'science city' em 2004. O autor conclui o texto sugerindo uma mudança nas políticas públicas de financiamento, visto que 
"mudanças no regime de financiamento são a maneira mais eficiente de se alterar como as universidades se comportam" (GODDARD, 2009, p. 4).

Na América Latina, o livro Universidades e Desenvolvimento Regional: as Bases para a Inovação Competitiva, organizado por Serra (2018) e outros autores, se propõe a analisar o impacto regional da universidade no desenvolvimento econômico sob três eixos: terceira missão, qualidade educacional na promoção do crescimento econômico, eficiência e financiamento. O capítulo do livro intitulado $O$ engajamento social como motor do desenvolvimento regional: a contribuição das universidades latinoamericanas, escrito por Mora et al. (2018), destaca a importância do engajamento social da universidade:

Pode-se dizer que a terceira missão ressurgiu e incluiu novas formas de interação entre as principais missões das universidades, mostrando que chegou a hora de se reconhecer um novo contrato social entre as universidades e as sociedades; de se aprender a fomentar e promover essas interações por meio de parcerias e ações colaborativas (MORA et al., 2018, p. 126).

Um estudo realizado pelos autores com 28 universidades da Argentina, Brasil, Chile, Colômbia, México, Paraguai e Peru buscou identificar quais atividades ligadas à terceira missão tem sido desenvolvidas por elas. Tais atividades foram divididas em três categorias (i) transferência de tecnologia e inovação, (ii) educação continuada e (iii) engajamento social. Os resultados mostram que a transferência de conhecimento (categoria i) é a mais desigual. Existem universidades que apresentam grande dimensão de interações nessa categoria, tais como a Universidade de São Paulo, a Universidade Católica do Chile e a Universidade Nacional Autônoma do México, enquanto outras - geralmente mais orientadas para o ensino - possuem fraca estrutura de transferência. $\mathrm{O}$ ensino continuado apresenta boa dimensão em todas as universidades. O engajamento social, porém, é a categoria mais perceptível, conforme mostra a Figura 1.

Figura 1 - Modelo de interação entre as universidades latino-americanas e a sociedade

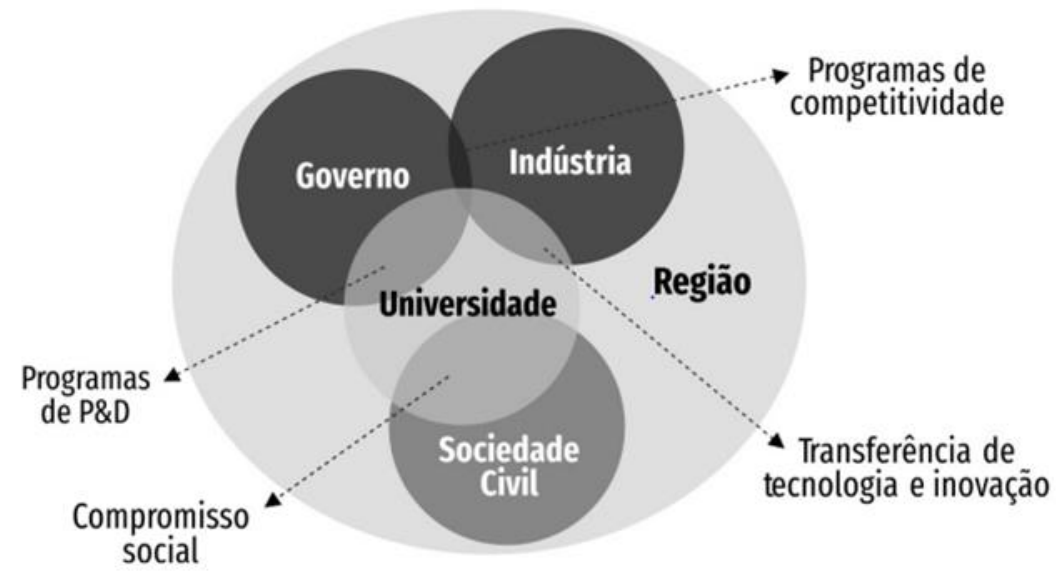

Fonte: MORA et al., 2018, p. 13

Cada círculo na Figura 3 mostra as relações entre universidade, indústria, governo e sociedade civil. A dimensão das interações no contexto latino-americano está representada pelas intersecções entre os círculos. Portanto, observa-se que a menor dimensão é a da interação universidade-indústria, seguida por universidade-governo. Conforme relembram os autores, essa característica é "um resultado direto de problemas estruturais numa região marcada por uma profunda instabilidade social e econômica [...]" (MORA et al., 2018, p. 136).

Pensando no papel da universidade em um sentido mais amplo e não apenas no seu papel como fornecedora de conhecimento para empresas orientadas pelo lucro, os autores Marcellino, Rapini e Chiarini (2019) buscaram preencher a lacuna de estudos empíricos sobre as interações da universidade para além da interação universidade-empresa no Brasil. Eles utilizaram dados do Diretório de Grupos de Pesquisa do CNPq de 2014 e 2016 para identificar com quais agentes as universidades brasileiras interagem e, também, padrões regionais. 
Os principais resultados demonstram que as interações se concentram nas regiões Sul e Sudeste do país, mais especificamente nos estados de Minas Gerais, São Paulo, Rio de Janeiro e Rio Grande do Sul, onde se encontram, também, a maior parte dos grupos de pesquisa. Os mesmos padrões de interação encontrados para o Brasil são encontrados nessas duas regiões. Nas outras regiões, observa-se que as relações universidade-universidade são mais intensas. O Centro-Oeste é a região que possui o número maior de interações com agências do governo, enquanto o Sul e o Norte possuem mais colaboração com cooperativas. Analisando mais a fundo somente os 5 maiores grupos de pesquisa de cada região, observase que os tipos de interação dentro da mesma área do conhecimento e da mesma região são diferentes entre eles. Além disso, observa-se que as interações dos 5 grupos não são restritas a empresas. Os grupos interagem, também, com cooperativas, o governo e outras universidades. Os autores concluem o artigo enfatizando a necessidade de os policy makers considerarem outros tipos de interação, não apenas universidade-empresa, visto que estas ocorrem e podem ser benéficas para a sociedade.

No contexto desta literatura, na próxima seção, são apresentados os dados de interação universidade-sociedade no Rio Grande do Sul. Tal análise busca demonstrar características gerais da interação universidade-sociedade, tais como principais parceiros e formas de interação.

\section{Metodologia}

Os dados utilizados na análise são oriundos do Diretório dos Grupos de Pesquisa (DGP), base de dados criada em 1990 pelo Conselho Nacional de Desenvolvimento Científico e Tecnológico (CNPq). Tais dados são disponibilizados na forma de Censos. Neste trabalho vale-se do último Censo disponível, de 2016.

A base organiza informações dos grupos de pesquisa brasileiros coletados bianualmente, através de um questionário enviado aos líderes desses grupos. O questionário coleta, entre outros dados, as seguintes informações:

a) Nome do grupo de pesquisa

b) Instituição à qual o grupo está ligado;

c) Localização da instituição;

d) Área de conhecimento do grupo;

e) Nome da instituição parceira;

f) Tipo de relação estabelecida com o parceiro.

Os grupos de pesquisa informados no Censo do DGP indicam se estabelecem parcerias ou não. Quando essas ocorrem, o líder do grupo informa o tipo de parceiro com quem interagem. Nesse artigo, os parceiros foram classificados a partir das categorias propostas por Marcellino, Rapini e Chiarini (2019), conforme apresentado no Quadro 1. Para simplificar a análise, realizou-se o agrupamento de algumas categorias, como Hospital $(12+13)$ e Outros $(4+8+14+15)$.

Quadro 1 - Categorias dos parceiros.

\begin{tabular}{|c|l|r|l|}
\hline 1 & Governo & 9 & Instituições - Organismos estrangeiros \\
\hline 2 & $\begin{array}{l}\text { Universidades, faculdades e institutos de } \\
\text { pesquisa }\end{array}$ & 10 & Empresa pública \\
\hline 3 & $\begin{array}{l}\text { Associações, Sindicatos, Sistema S, Sebrae, } \\
\text { Organizações }\end{array}$ & 11 & Fundação \\
\hline 4 & Cooperativas & 12 & Hospital \\
\hline 5 & Universidades e IPTs* estrangeiras & 13 & Hospital estrangeiro \\
\hline 6 & Empresas nacionais & 14 & Governo estrangeiro \\
\hline 7 & Empresas multinacionais & 15 & Associação estrangeira \\
\hline 8 & Banco & & \\
\hline
\end{tabular}

Fonte: Marcellino, Rapini e Chiarini, 2019, p. 6.

Nota: (*) Institutos de Pesquisa Tecnológica.

No que tange ao tipo de relação estabelecida, o diretório propõe quatorze diferentes categorias. O 
líder do grupo de pesquisa pode escolher até três para cada parceiro, sem ordem de hierarquia. Para simplificação da análise, os relacionamentos bilaterais foram agrupados em um. As nove categorias resultantes são apresentadas no Quadro 2.

Quadro 2 - Tipos de interação entre grupos de pesquisa e parceiros externos.

\begin{tabular}{|l|l|}
\hline 1 & Consultoria \\
\hline 2 & $\begin{array}{l}\text { Atividades de engenharia não-rotineira (inclusive o desenvolvimento de protótipo, cabeça de série ou } \\
\text { planta-piloto)* }\end{array}$ \\
\hline 3 & Desenvolvimento de software* \\
\hline 4 & $\begin{array}{l}\text { Fornecimento de insumos materiais para as atividades do parceiro sem vinculação a um projeto específico } \\
\text { de interesse mútuo }\end{array}$ \\
\hline 5 & Pesquisa científica com considerações de uso imediato dos resultados \\
\hline 6 & Pesquisa científica sem considerações de uso imediato dos resultados \\
\hline 7 & Transferência de tecnologia* \\
\hline 8 & Treinamento (incluindo cursos e treinamento "em serviço") * \\
\hline 9 & Outros \\
\hline
\end{tabular}

Fonte: CNPq, 2016.

Nota: (*) possibilidade de relacionamento bilateral.

Assim, a partir dessas informações, realizou-se uma análise descritiva, buscando compreender aspectos gerais das interações dos grupos de pesquisa ligados a universidades do RS com outros agentes da sociedade. Cabe lembrar que os grupos de pesquisa contidos na base de dados do DGP / CNPq podem estar vinculados a diferentes instituições. Porém, como o foco deste trabalho é a análise das interações das universidades, foram filtrados para a análise apenas os grupos de pesquisa ligados a universidades e faculdades. Os resultados são apresentados na próxima seção.

\section{Análise descritiva dos dados}

Foram analisadas 31 universidades e faculdades sul riograndenses e 3.178 grupos de pesquisa, ligados a elas, conforme detalhado por área do conhecimento na Tabela 1. Da contagem total de grupos, cerca de $35,27 \%$ possuem interação com parceiros externos.

Tabela 1- Grupos de pesquisa por área do conhecimento no RS

\begin{tabular}{lccc}
\hline & Grupos no total (A) & Grupos com interação (B) & (B)/(A) \\
\hline Ciências agrárias & 286 & 146 & $51,05 \%$ \\
\hline Ciências biológicas & 290 & 135 & $46,55 \%$ \\
\hline Ciências da saúde & 522 & 155 & $29,69 \%$ \\
\hline Ciências exatas e da terra & 394 & 172 & $43,65 \%$ \\
\hline Ciências humanas & 659 & 206 & $31,26 \%$ \\
\hline Ciências sociais aplicadas & 558 & 134 & $24,01 \%$ \\
\hline Engenharias & 253 & 123 & $48,62 \%$ \\
\hline Linguística, letras e artes & 213 & 49 & $23,00 \%$ \\
\hline Outra & 3 & 1 & $33,33 \%$ \\
\hline Total & $\mathbf{3 1 7 8}$ & $\mathbf{1 1 2 1}$ & \\
\hline
\end{tabular}

Fonte: elaborado pela autora, com base nos dados do Censo 2016 do DGP/CNPq (2020).

Apesar das Ciências Humanas, das Ciências Exatas e das Ciências da Saúde possuírem o maior volume de grupos interativos, as áreas do conhecimento que possuem a maior proporção de grupos interativos são as Ciências Agrárias (51,05\%), as Engenharias (48,62\%) e as Ciências Biológicas $(46,55 \%)$. Esse resultado vai ao encontro do estudo em nível nacional de Marcellino, Rapini e Chiarini 
(2019), que demonstrou que as áreas do conhecimento com maior razão "grupos com interação/grupos totais" no Brasil também são as Ciências Agrárias $(46,6 \%)$ e as Engenharias $(41,2 \%)$.

Na Tabela 2, observa-se que o número absoluto de grupos interativos é $64,4 \%$ maior em universidades públicas. Porém quando se compara a proporção de grupos interativos em relação ao total de grupos, as universidades públicas e privadas têm um padrão semelhante. A relação "grupos com interação/grupos totais" de universidades públicas é de $34,47 \%$, enquanto em universidades privadas esse valor é de $36,68 \%$.

Tabela 2 - Grupos com interação por tipo de universidade no RS.

\begin{tabular}{llll}
\hline & $\begin{array}{l}\text { Grupos no } \\
\text { total }\end{array}$ & $\begin{array}{l}\text { Grupos com } \\
\text { interação }\end{array}$ & $\begin{array}{l}\text { Grupos com interação/ } \\
\text { grupos totais }\end{array}$ \\
\hline Universidades públicas & 2022 & 697 & $34,47 \%$ \\
\hline Universidades privadas & 1156 & 424 & $36,68 \%$ \\
\hline Total & $\mathbf{3 1 7 8}$ & $\mathbf{1 1 2 1}$ & $\mathbf{3 5 , 2 7 \%}$ \\
\hline
\end{tabular}

Fonte: elaborado pela autora com base nos dados do Censo 2016 do DGP/CNPq (2020).

Considerando todas as universidades do RS, verifica-se que a universidade que ocupa o primeiro lugar no ranking daquelas com maior número de grupos interativos é a Universidade Federal do Rio Grande do Sul, com 322 grupos interativos, seguida pela Universidade Federal de Santa Maria (149 grupos), ambas universidades públicas. Em terceiro lugar está a universidade privada Pontifícia Universidade Católica do Rio Grande do Sul, com 134 grupos interativos.

Além da análise por área do conhecimento e por tipo de universidade, demonstrados nas tabelas anteriores, outro foco desse trabalho é identificar o tipo de parceiro com o qual os grupos de pesquisa possuem interação. Esses parceiros, conforme mencionado anteriormente, foram classificados em categorias.

A Tabela 3 apresenta o número total de agentes por categoria, com os quais os grupos de pesquisa interagiram, bem como o número total de interações registradas por agente. Em comparação com o Brasil, conforme demonstrado por Marcellino, Rapini e Chiarini (2019), percebe-se que a relação "interações/agente" no Rio Grande do Sul está bem abaixo da média nacional no mesmo período, cujo valor foi 7,7, enquanto no estado essa média foi de 1,99 .

Ao analisar cada categoria separadamente, é possível identificar que a empresa pública é o parceiro que possui a maior razão "interações/agente": 3,37 . Em segundo lugar estão as universidades e os Institutos de Ciência e Tecnologia (ICTs) com uma razão de 2,95. Em nível nacional, de acordo com Marcellino, Rapini e Chiarini (2019), as universidades e ICTs são o parceiro que apresenta maior razão "interações/agente" no mesmo período, totalizando 22,8. Em segundo lugar está o Governo com uma razão de 5,2 .

Tabela 3 - Interações por agente da sociedade.

\begin{tabular}{lccc}
\hline Categorias & Agentes & Interações & Interações/agente \\
\hline $\begin{array}{l}\text { Associações, sindicatos, sistema S, } \\
\text { Sebrae e organizações }\end{array}$ & 69 & 94 & 1,36 \\
\hline Empresa pública & 43 & 145 & 3,37 \\
\hline Empresas multinacionais & 82 & 110 & 1,34 \\
\hline Empresas nacionais & 308 & 370 & 1,20 \\
\hline Fundação & 38 & 86 & 2,26 \\
\hline Governo & 67 & 108 & 1,61 \\
\hline Hospital & 19 & 49 & 2,58 \\
\hline Universidades e IPTs estrangeiras & 353 & 486 & 1,38 \\
\hline $\begin{array}{l}\text { Universidades, faculdades e institutos } \\
\text { de pesquisa }\end{array}$ & 557 & 1645 & 2,95
\end{tabular}




\begin{tabular}{lccc}
\hline Outros & 47 & 62 & 1,32 \\
\hline Total & $\mathbf{1 5 8 3}$ & $\mathbf{3 1 5 5}$ & $\mathbf{1 , 9 9}$ \\
\hline
\end{tabular}

Fonte: elaborado pela autora, com base nos dados do Censo 2016 do DGP/CNPq (2020).

Apesar do maior número de parceiros dos grupos de pesquisa gaúchos se concentrarem em universidades e empresas, existe considerável interação com outros agentes da sociedade, como associações, fundações, governo e hospitais.

A categoria "Associações, sindicatos, sistema S, Sebrae e organizações" engloba uma variedade de parceiros. Nela estão, por exemplo, o Instituto do Câncer Infantil, uma associação que visa assistir crianças e adolescentes com câncer. Há, também, o Serviço Brasileiro de Apoio às Micro e Pequenas Empresas (SEBRAE), uma associação sem fins lucrativos de capacitação e promoção do desenvolvimento, voltado para pequenos negócios. Além disso, constam, também, os sindicatos e as organizações do Sistema S, responsável por promover educação e desenvolvimento para os setores da indústria, comércio e serviços.

Na categoria "Fundação" estão importantes instituições voltadas à proteção do meio-ambiente, como a Fundação Estadual de Proteção Ambiental Henrique Luís Roessler (em Porto Alegre, RS), o Instituto Baleia Jubarte (em Caravelas, BA) e a Fundação O Boticário de Proteção à Natureza (Curitiba, PR). Também estão instituições de incentivo à arte, como a Fundação Nacional de Artes (Rio de Janeiro, RJ) e a Fundação Municipal de Artes de Montenegro (Montenegro, RS).

Em "governo" encontram-se órgãos governamentais municipais, estaduais e federais. Alguns deles são: Departamento Municipal de Águas e Esgotos de Porto Alegre, Secretaria de Educação do Estado do Rio Grande do Sul, Agência Nacional de Transportes Terrestres, Departamento Nacional de Produção Mineral, entre outros. São órgãos que exercem funções fundamentais para a qualidade de vida da população.

Os hospitais nacionais e estrangeiros foram agrupados em apenas uma categoria para simplificar a análise, visto que havia apenas um hospital estrangeiro parceiro: Liverpool Heart and Chest Hospital. Os outros hospitais são nacionais e em sua maioria localizados em Porto Alegre, tais como: Hospital de Clínicas de Porto Alegre, Hospital Mãe de Deus e Hospital Nossa Senhora da Conceição.

Já os parceiros caracterizados como empresa estão subdivididos em três categorias: Empresa pública, Empresa multinacional e Empresa nacional. Na categoria "Empresa pública" estão gigantes como a Petrobrás e a Companhia Energética de Minas Gerais (CEMIG) ${ }^{1}$, bem como empresas regionais, como a Empresa de Trens Urbanos de Porto Alegre e Empresa Pública de Transporte e Circulação (EPTC). Em 'Empresas multinacionais' estão companhias que realizam atividades em diversos países, tais como a Dell, a Samsung e a Motorola, do ramo de tecnologia e a PepsiCo, do ramo alimentício. Também estão algumas empresas nacionais, que também atuam em outros países, como a Companhia Vale do Rio Doce, empresa de mineração, e a Gerdau, indústria de aço fundada no Rio Grande do Sul. Na categoria de 'Empresas nacionais', encontram-se empresas brasileiras de setores diversos, como agroindustrial, têxtil e calçadista e alimentício. Alguns exemplos de empresas nessa categoria são: Grendene, Calçados Azaleia, Sultêxtil Indústria e Tecidos, TANAC S/A e Safra Agronegócios.

As 'Universidades, faculdades e institutos de pesquisa', divididos em nacionais e estrangeiros, são a categoria com maior volume de interações em termos absolutos. Entre as nacionais, as principais são do próprio estado, devido às facilidades advindas da proximidade geográfica. Entre as internacionais, estão universidades de diversos países, como: Universidad de la República Uruguay, Universidad Autónoma de Madrid, Universidade de Lisboa e Harvard University.

$\mathrm{Na}$ categoria "Outros", foram agrupadas as "Cooperativas", "Bancos" e "Associações estrangeiras", por causa do baixo volume de parceiros nessas categorias. As cooperativas, em sua maioria, estão ligadas ao setor agropecuário, tais como: Cooperativa Agroindustrial Consolata e Cooperativa Arrozeira Extremo Sul. Os bancos são apenas dois: Banco Santander S.A. e Caixa Econômica Federal. Já em associações estrangeiras estão organizações importantes, como: Organização Mundial da Saúde e

\footnotetext{
${ }^{1}$ Essas empresas são classificadas como sociedades de economia mista. Para que não se criasse mais uma categoria, preferiu-se classificá-las como empresas públicas.
} 


\section{Organização das Nações Unidas.}

A Figura 2 é um mapa de calor que mostra a concentração geográfica dos parceiros dos grupos de pesquisa do Rio Grande do Sul, descritos anteriormente. Os pontos mais escuros mostram onde existe o maior número de parceiros. Observa-se que $38,34 \%$ deles se concentram no próprio estado.

Figura 2 - Concentração Geográfica dos Parceiros.

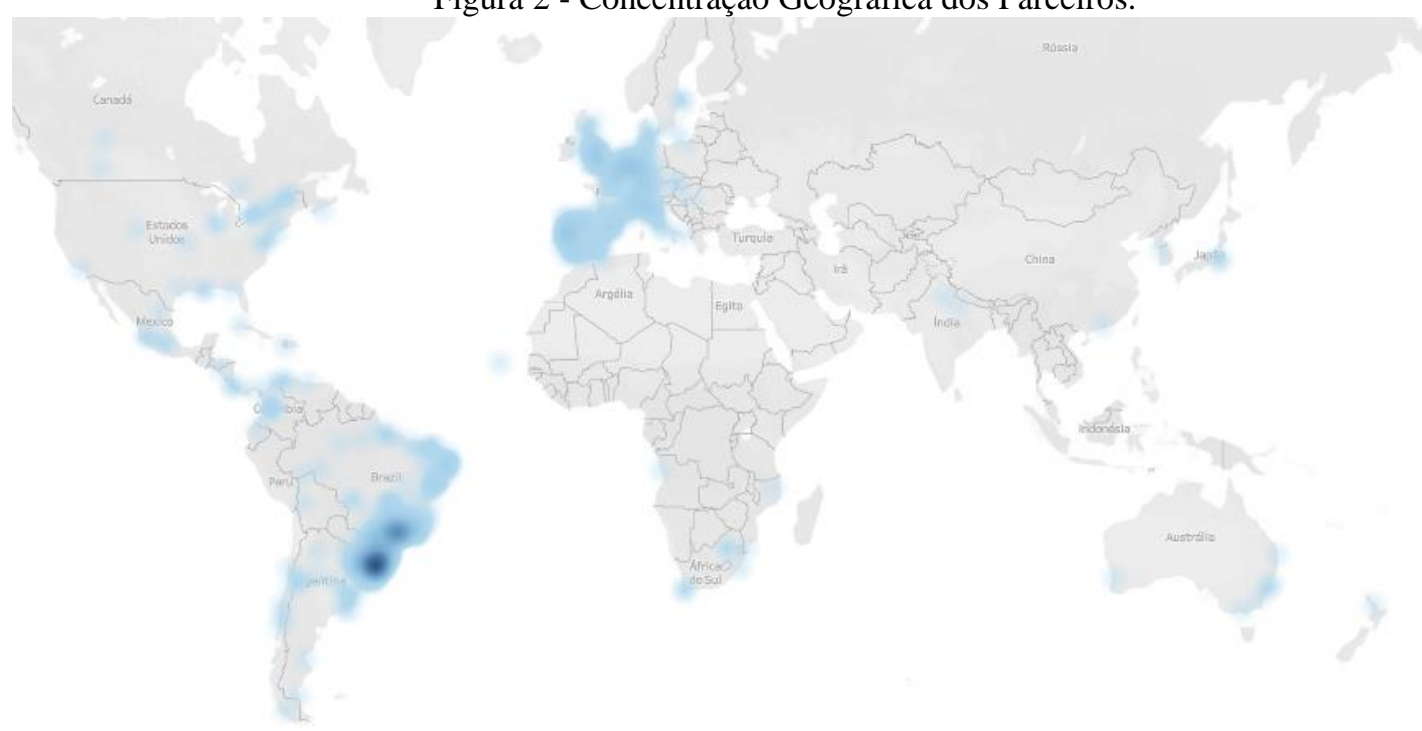

Fonte: elaborado pela autora, com base nos dados do Censo 2016 do DPG/CNPq (2020).

Os parceiros internacionais constituem cerca de $24,2 \%$ do total e se concentram na Europa e nas Américas. Eles são, em grande maioria, universidades e IPTs estrangeiras e, em minoria, empresas multinacionais. O restante dos parceiros se concentra em nível nacional, principalmente no eixo sulsudeste, o que demonstra a influência regional da universidade gaúcha. A Figura 3 demonstra a distribuição geográfica dos principais parceiros dentro do Brasil.

Figura 3 - Distribuição Geográfica quando os parceiros são Universidades e Empresas.

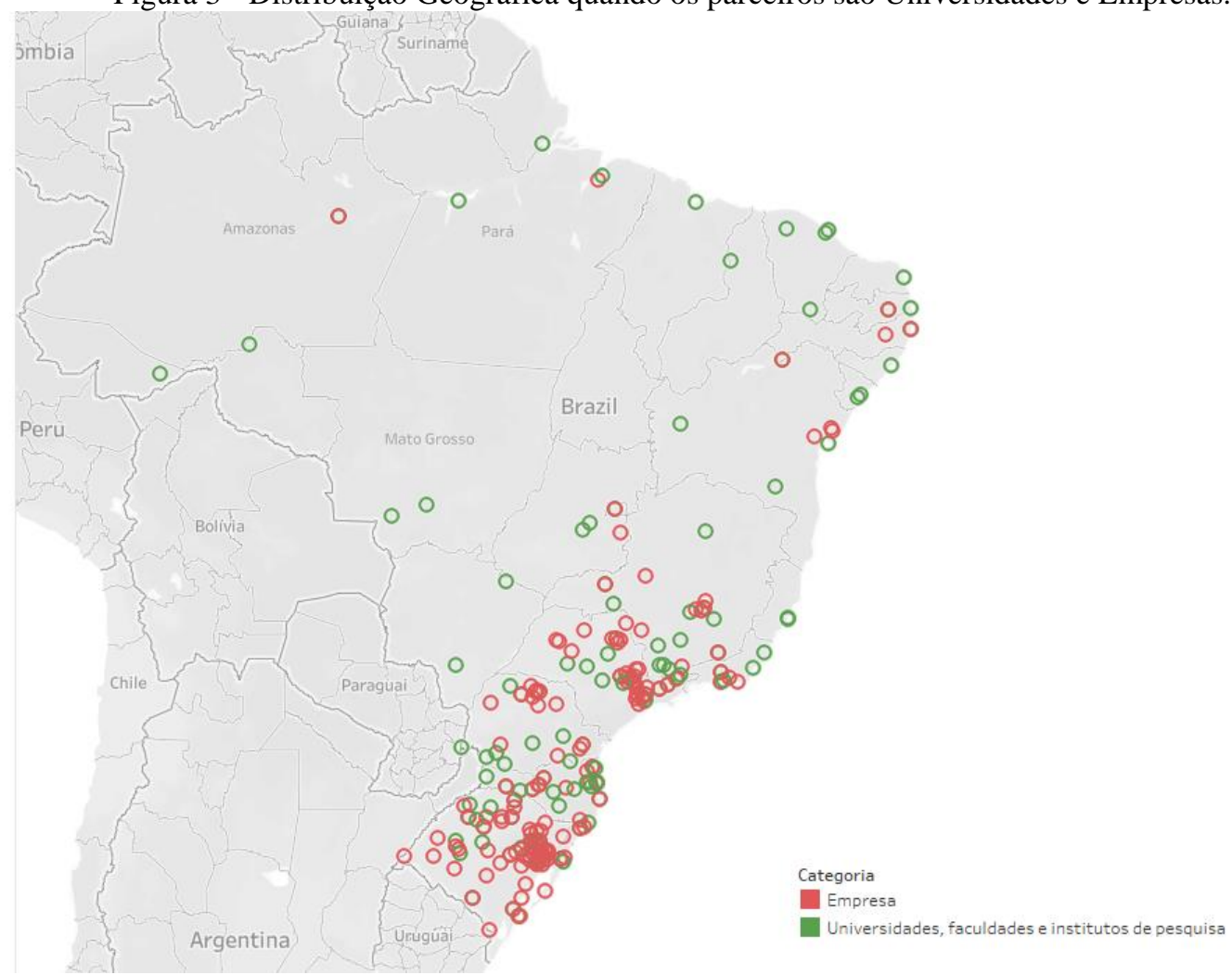

Fonte: elaborado pela autora, com base nos dados do Censo 2016 do DPG/CNPq (2020). 
As empresas nacionais, multinacionais e públicas foram agrupadas em "Empresa" para facilitar a visualização. Observa-se que as universidades estão mais dispersas em todo o país, enquanto as empresas apresentam maior concentração no eixo sul-sudeste. De fato, essas são as regiões mais desenvolvidas e dinâmicas do país, onde se encontram as sedes das maiores firmas.

Após identificados os parceiros e suas características, realizou-se um cruzamento entre a categoria do parceiro e o tipo de universidade a qual pertence o grupo de pesquisa. Os resultados são demonstrados na Tabela 4.

Tabela 4 - Interações por tipo de universidade e categoria do parceiro.

\begin{tabular}{|c|c|c|c|c|c|c|}
\hline \multirow[b]{2}{*}{ Categorias } & \multicolumn{3}{|c|}{ Universidade Pública } & \multicolumn{3}{|c|}{ Universidade Privada } \\
\hline & Agentes & Interações & $\begin{array}{l}\text { Interações/ } \\
\text { agente }\end{array}$ & Agentes & Interações & $\begin{array}{c}\text { Interações/ } \\
\text { agente }\end{array}$ \\
\hline $\begin{array}{l}\text { Associações, } \\
\text { sindicatos, sistema } \\
\text { S, Sebrae e } \\
\text { organizações }\end{array}$ & 41 & 53 & 1,29 & 36 & 41 & 1,14 \\
\hline Empresa pública & 33 & 106 & 3,21 & 22 & 39 & 1,77 \\
\hline $\begin{array}{l}\text { Empresas } \\
\text { multinacionais }\end{array}$ & 62 & 75 & 1,21 & 26 & 35 & 1,35 \\
\hline Empresas nacionais & 217 & 257 & 1,18 & 104 & 113 & 1,09 \\
\hline Fundação & 32 & 58 & 1,81 & 14 & 28 & 2,00 \\
\hline Governo & 46 & 69 & 1,50 & 27 & 39 & 1,44 \\
\hline Hospital & 9 & 28 & 3,11 & 13 & 21 & 1,62 \\
\hline Outros & 36 & 45 & 1,25 & 14 & 17 & 1,21 \\
\hline $\begin{array}{l}\text { Universidades e } \\
\text { IPTS estrangeiras }\end{array}$ & 231 & 287 & 1,24 & 158 & 199 & 1,26 \\
\hline $\begin{array}{l}\text { Universidades, } \\
\text { faculdades e } \\
\text { institutos de } \\
\text { pesquisa }\end{array}$ & 408 & 1041 & 2,55 & 273 & 604 & 2,21 \\
\hline Total & 1115 & 2019 & 1,81 & 687 & 1136 & 1,65 \\
\hline
\end{tabular}

Fonte: elaborado pela autora, com base nos dados do Censo 2016 do DPG/CNPq (2020).

Universidades públicas possuem maior número absoluto de interações, o que corrobora o fato desta possuir maior número absoluto de grupos de pesquisa, conforme observado na Tabela 2 . Além disso, a relação "interações/agente" na universidade pública é maior para a grande maioria das categorias de parceiros, com exceção de Empresas multinacionais, Fundação e Universidades e IPTs estrangeiras, indicando que as universidades particulares possuem uma intensidade maior de interações com o exterior.

Com relação ao tipo de parceiro com o qual cada tipo de universidade mais interage, percebe-se que também não existe grande diferença: tanto os grupos de pesquisa de universidades públicas quanto das privadas interagem principalmente com Universidades e ICTS nacionais e estrangeiras e Empresas nacionais. Uma diferença marcante está na interação com Empresas públicas, que é proporcionalmente maior entre as universidades públicas. Uma hipótese que pode explicar esse dado é a proximidade entre instituições administradas pelo Estado. Outra diferença está na interação com os Hospitais, que é mais intensa em universidades públicas, provavelmente devido ao maior número de hospitais universitários ligados a instituições de ensino públicas.

A seguir, realizou-se um cruzamento entre a área do conhecimento e a categoria de parceiro, conforme demonstra o Gráfico 3. O tamanho do círculo representa a quantidade de interações que ocorre entre a categoria (linhas) e a grande área (colunas); quanto maior o círculo, maior a quantidade de interações. 


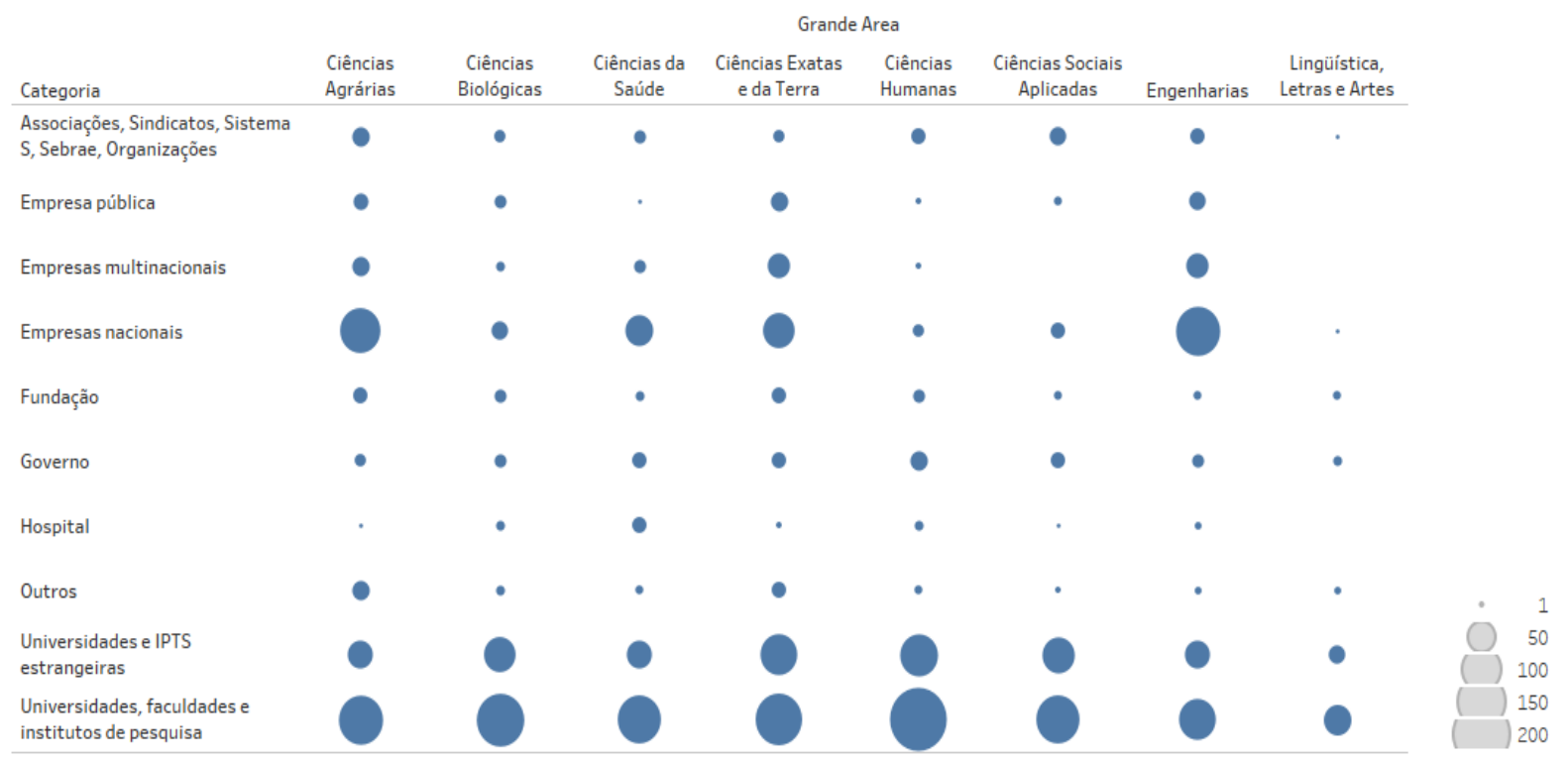

Gráfico 1 - Número de interações por grande área e categoria do parceiro. Fonte: elaborado pela autora, com base nos dados do Censo 2016 do DGP/CNPq (2020).

Percebe-se que as Universidades, faculdades e institutos de pesquisa, tanto nacionais quanto estrangeiras, são os principais parceiros dos grupos de pesquisa de todas as áreas do conhecimento. Outro parceiro de destaque são as Empresas nacionais, que interagem principalmente nas Ciências Agrárias e Engenharias. Cabe lembrar que essas duas grandes áreas são as que possuem maior proporção de grupos interativos, conforme o Gráfico 1. Além disso, a relação "interações/agente" nas Empresas nacionais é mais alta do que em Universidades, faculdades e institutos de pesquisa. Pode-se dizer, portanto, que as interações com Universidades são mais frequentes, enquanto as interações com Empresas nacionais são mais intensas.

Outro dado relevante coletado no questionário aplicado pelo DGP/CNPq, é o tipo de relação estabelecida entre o grupo de pesquisa e o parceiro. São, ao todo, 14 categorias diferentes propostas pelo Diretório, porém neste trabalho consideramos as relações bilaterais como apenas uma, totalizando 9 tipos. Os líderes dos grupos podem escolher até três tipos de relação para cada parceiro, sem ordem de hierarquia. Os resultados são demonstrados no Gráfico 4. Quanto maior o tamanho do círculo, maior a ocorrência.

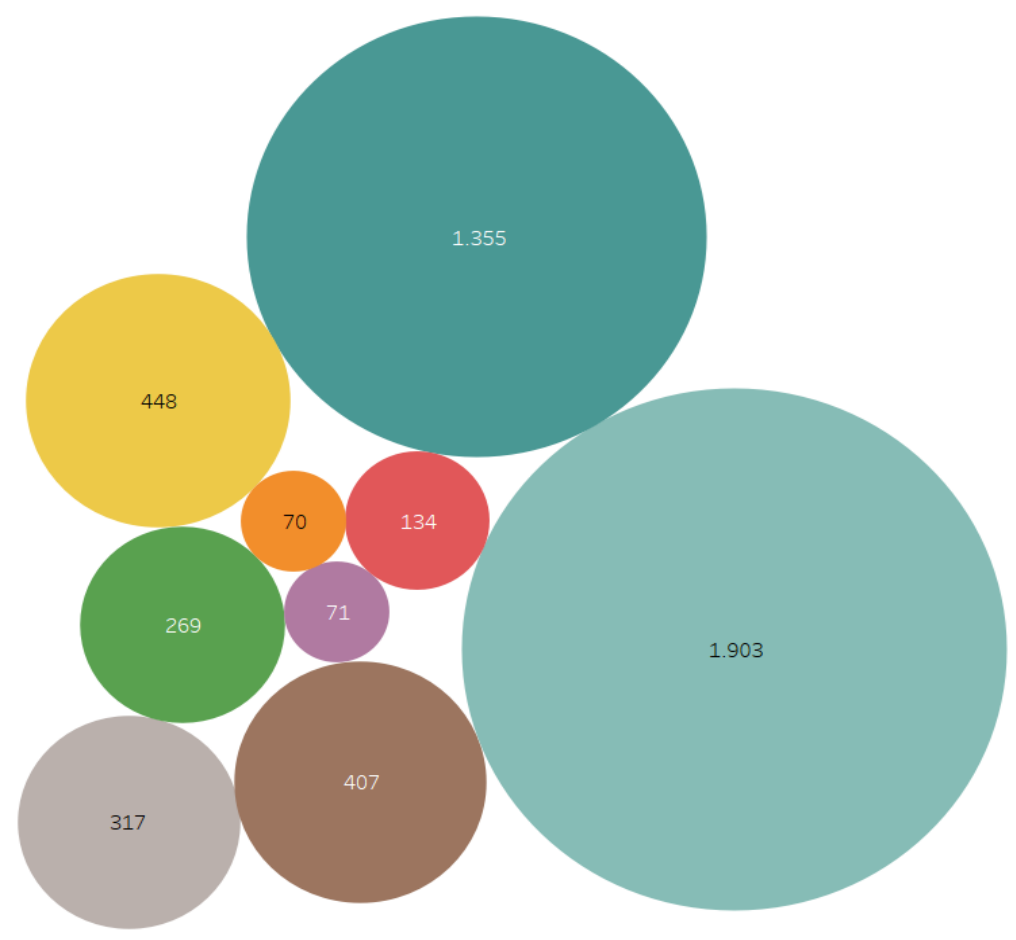




\section{tipo de relação \\ Atividades de engenharia não-rotineira (inclusive o desenvolvimento de protótipo, cabeça de série ou planta-piloto) \\ Consultoria \\ Desenvolvimento de software \\ Fornecimento de insumos materiais para as atividades do parceiro sem vinculação a um projeto específico de interesse mútuo \\ Outros \\ Pesquisa científica com considerações de uso imediato dos resultados \\ Pesquisa científica sem considerações de uso imediato dos resultados \\ Transferência de tecnologia \\ Treinamento (incluindo cursos e treinamento "em serviço"}

Gráfico 2 - Número de ocorrências do tipo de interação. Fonte: elaborado pela autora, com base nos dados do Censo 2016 do DGP/CNPq (2020).

Esse gráfico demonstra, portanto, que o tipo de interação mais frequente praticada pelos grupos de pesquisa do Rio Grande do Sul é a pesquisa científica sem considerações de uso imediato; o que pode ser explicado pelo significativo volume de interações ocorrer com instituições de ensino e pesquisa. Em segundo lugar, está a pesquisa científica com considerações de uso imediato. Em seguida estão a transferência tecnológica e o treinamento. Os tipos de interação podem ser explicados qualitativamente pela categoria dos parceiros, que se concentram em Universidades, faculdades e institutos de pesquisa e Empresas nacionais. As empresas nacionais parceiras são, em sua maioria, de setores tradicionais como o calçadista, têxtil, bem como do setor agropecuário. Isso explica a baixa ocorrência de interações do tipo desenvolvimento de software e atividades de engenharia não-rotineira, que estão ligados a empresas de maior complexidade tecnológica.

O Gráfico 3, a seguir, complementa a análise feita a partir do Gráfico 2, pois realizou-se o cruzamento entre o tipo de relação estabelecida pelo grupo de pesquisa e as categorias dos seus parceiros. A partir dessa análise, observa-se que, o tipo de interação mais frequente entre grupos de pesquisa e universidades parceiras é a pesquisa científica sem considerações de uso imediato dos resultados (47,66\%), enquanto entre os grupos e empresas nacionais parceiras, o tipo de interação mais frequente é a pesquisa científica com considerações de uso imediato dos resultados $(32,80 \%)$ seguido de transferência tecnológica $(17,77 \%)$. Isso demonstra o caráter mais aplicado das pesquisas quando os parceiros são empresas.

\begin{tabular}{|c|c|c|c|c|c|c|c|c|c|c|c|}
\hline & $\begin{array}{c}\text { Associações, } \\
\text { Sindicatos, } \\
\text { Sistema S, } \\
\text { Sebrae, Organ.. }\end{array}$ & $\begin{array}{l}\text { Empresa } \\
\text { pública }\end{array}$ & $\begin{array}{c}\text { Empresas } \\
\text { multinacionais }\end{array}$ & $\begin{array}{l}\text { Empresas } \\
\text { nacionais }\end{array}$ & Fundação & Governo & Hospital & Outros & $\begin{array}{l}\text { Universidades } \\
\text { elPTS } \\
\text { estrangeiras }\end{array}$ & $\begin{array}{l}\text { Universidades, } \\
\text { faculdadese } \\
\text { institutos de } \\
\text { pesquisa }\end{array}$ & \\
\hline $\begin{array}{l}\text { Atividades de } \\
\text { engenharia nâo-rotine.. }\end{array}$ & & & • & • & & & & & & $\cdot$ & \\
\hline Consultoria & • & & • & • & & - & & • & • & $\bullet$ & \\
\hline $\begin{array}{l}\text { Desenvolvimento de } \\
\text { software }\end{array}$ & & - & & - & & & & & - & • & \\
\hline $\begin{array}{l}\text { Fornecimento de } \\
\text { insumos materiais par.. }\end{array}$ & • & • & • & $\bullet$ & & - & & - & - & • & \\
\hline Outros & - & • & • & $\bullet$ & • & - & - & - & $\bullet$ & ○ & \\
\hline $\begin{array}{l}\text { Pesquisa cientifica com } \\
\text { considerações de uso i... }\end{array}$ & $\bullet$ & - & - & 0 & - & • & - & - & • & & \\
\hline $\begin{array}{l}\text { Pesquisa científica sem } \\
\text { consideraçôes de uso i.. }\end{array}$ & • & • & - & - & • & • & - & - & 0 & & $\begin{array}{l}200 \\
\quad 400\end{array}$ \\
\hline $\begin{array}{l}\text { Transferência de } \\
\text { tecnologia }\end{array}$ & • & - & - & $\bullet$ & & - & & • & - & - & $\begin{array}{l}600 \\
800\end{array}$ \\
\hline $\begin{array}{l}\text { Treinamento (incluindo } \\
\text { cursos e treinamento .. }\end{array}$ & - & • & & $\bullet$ & $\cdot$ & • & & • & $\bullet$ & • & $\begin{array}{l}1.000 \\
1.160\end{array}$ \\
\hline
\end{tabular}

Gráfico 3 - Número de ocorrências do tipo de interação por categoria de parceiro. Fonte: elaborado pela autora, com base nos dados do Censo 2016 do DGP/CNPq (2020).

Nas demais categorias, também é mais forte o tipo de interação voltado para a pesquisa. A pesquisa científica sem considerações de uso imediato dos resultados prevalece no Governo $(29,73 \%)$, nas Fundações (40,98\%) e nas Empresas públicas (34,50\%). Já a pesquisa científica com considerações de uso imediato representa uma maior porcentagem do total de interações em Associações e Sindicatos $(27,27 \%)$, Empresas multinacionais $(31,25 \%)$, Hospitais $(35,90 \%)$ e Outros $(27,84 \%)$. Secundariamente, a 
transferência tecnológica pode ser considerada representativa em Associações e sindicatos $(20,54 \%)$ e Empresas multinacionais $(14,06 \%)$.

Após analisar de maneira agregada os grupos de pesquisa do Rio Grande do Sul, selecionou-se 5 grupos de pesquisa das universidades públicas e privadas, com maior número de parceiros para uma análise individualizada. A Tabela 5 apresenta a universidade a qual o grupo está vinculado, o nome do grupo, o número de parceiros do grupo, o seu ano de criação, a área do conhecimento e as categorias dos seus parceiros.

As áreas do conhecimento que se destacam entre os 5 grupos são as Ciências Agrárias e as Ciências Biológicas. Percebe-se que entre os grupos das universidades públicas, a variedade de parceiros é maior, enquanto os parceiros das universidades privadas se concentram em outras universidades e ICTs. Independente disso, destaca-se que as interações desses grupos de pesquisa ocorrem ainda com diversos atores da sociedade: Empresas, Associações, Hospitais, Governo e Fundações. 
Tabela 5 - Top5 grupos de pesquisa em número de parceiros por universidades públicas e privadas.

\begin{tabular}{|c|c|c|c|c|c|c|}
\hline & Instituição* & Grupo de pesquisa & $\begin{array}{l}\text { Número de } \\
\text { parceiros }\end{array}$ & $\begin{array}{l}\text { Ano de } \\
\text { criação }\end{array}$ & Área do conhecimento & Categoria dos parceiros \\
\hline \multirow{5}{*}{ 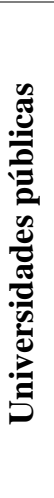 } & UFRGS & Bioengenharia de Alimentos & 27 & 2002 & Ciências Agrárias & $\begin{array}{l}\text { Universidades e ICTs, Governo, } \\
\text { Empresas e Fundação }\end{array}$ \\
\hline & UFSM & Microtoxinas/Microtoxicoses e Saúde Pública & 26 & 1998 & Ciências Agrárias & $\begin{array}{l}\text { Empresas, Associações e } \\
\text { Hospitais }\end{array}$ \\
\hline & UFRGS & $\begin{array}{l}\text { Avaliação, desenvolvimento e implementação } \\
\text { de terapias para doenças genéticas }\end{array}$ & 20 & 2008 & Ciências Biológicas & $\begin{array}{l}\text { Universidades e ICTs, Hospitais e } \\
\text { Associações }\end{array}$ \\
\hline & UFRGS & $\begin{array}{l}\text { Pesquisa, ação e colaboração em educação e } \\
\text { ensino da saúde - EducaSaúde }\end{array}$ & 19 & 2005 & Ciências Humanas & $\begin{array}{l}\text { Universidades e ICTs, Governo, } \\
\text { Hospitais e Associações }\end{array}$ \\
\hline & UFRGS & Concepção de Circuitos Integrados & 18 & 1983 & Ciências Exatas e da Terra & Universidades e ICTs e Empresas \\
\hline \multirow{5}{*}{ 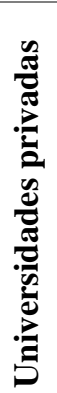 } & UNISINOS & PROCESSOCOM: processos comunicacionais & 19 & 2002 & Ciências Sociais Aplicadas & Universidades e ICTs \\
\hline & PUCRS & UNIVERSITAS/RIES & 16 & 1997 & Ciências Humanas & Universidades e ICTs \\
\hline & UNISINOS & Ornitologia & 14 & 1982 & Ciências Biológicas & Universidades e ICTs \\
\hline & ULBRA & TOXIGEN - investigação de toxicidade genética & 13 & 2010 & Ciências Biológicas & Universidades e ICTs e Fundação \\
\hline & URI & $\begin{array}{l}\text { Engenharia de Processos nas Indústrias Química } \\
\text { e de Alimentos }\end{array}$ & 11 & 2001 & Ciências Agrárias & $\begin{array}{l}\text { Universidades e ICTs, Empresas e } \\
\text { Associações }\end{array}$ \\
\hline
\end{tabular}

Fonte: elaborado pela autora com base nos dados do Censo 2016 do DGP/CNPq (2020).

Nota: (*) Universidade Federal do Rio Grande do Sul (UFRGS), Universidade Federal de Santa Maria (UFSM), Universidade do Vale do Rio dos Sinos (UNISINOS),

Pontifícia Universidade Católica do Rio Grande do Sul (PUCRS), Universidade Luterana do Brasil (ULBRA), Universidade Regional Integrada do Alto Uruguai e das

Missões (URI). 


\section{Considerações finais}

Esse artigo teve como objetivo contribuir para o estudo das interações universidade-sociedade, num sentido amplo, e não apenas universidade-empresa. Diversos autores já concluíram que a interação universidade-empresa em países como o Brasil é limitada devido ao baixo investimento em P\&D nas empresas e à ausência de indústrias de tecnologia de ponta. Mora et al. (2018) argumentam que as universidades da América Latina não conseguiram reproduzir os modelos europeu e estado-unidense, apesar de terem tentado. Fatores como a péssima distribuição de renda e as instabilidades políticas e econômicas impedem a difusão do conhecimento tal como ocorre nos países desenvolvidos.

A partir da análise dos dados de interações entre grupos de pesquisa ligados a universidades no Rio Grande do Sul e suas instituições parceiras, identificou-se que Empresas Públicas e as Universidades, faculdades e institutos de pesquisa são os parceiros com os quais os grupos possuem maior razão "interações/agente". Identificou-se, também, que a proporção "interações/agente" no estado é muito mais baixa que a média nacional. Apesar disso, os dados corroboram a existência de interação entre os grupos de pesquisa gaúchos e um conjunto diverso de atores, como o governo, hospitais, associações e fundações, e não apenas organizações voltadas para o lucro. Quanto à distribuição geográfica dos parceiros, existe grande concentração no eixo sul-sudeste, sendo que 38,34\% dos parceiros se encontram localizados no próprio estado, demonstrando a influência regional das universidades do RS.

Um cruzamento entre as categorias dos parceiros e o tipo de universidade a qual pertence o grupo de pesquisa mostrou que as universidades privadas possuem interações mais intensas com multinacionais e Universidades e IPTs estrangeiras, enquanto as universidades públicas interagem mais com Empresas públicas e Hospitais. Realizou-se, também, um cruzamento entre as categorias dos parceiros e a área do conhecimento do grupo de pesquisa. Observou-se que as Universidades e ICTs são os principais parceiros em todas as áreas do conhecimento. As Empresas Nacionais destacam-se na interação com os grupos de pesquisa das Ciências Agrárias e Engenharias.

Finalmente, analisou-se o tipo de relação estabelecida entre os grupos de pesquisa e seus parceiros. Os resultados demonstram que o tipo de interação mais frequente é a pesquisa científica sem considerações de uso imediato; em segundo lugar está a pesquisa científica com considerações de uso imediato. A primeira prevalece quando os parceiros são Universidades e ICTs, enquanto a segunda prevalece quando os parceiros são Empresas no geral. Em contrapartida, os tipos de interação que menos ocorrem são o desenvolvimento de software e atividades de engenharia não-rotineira. Isso se explica pela baixa complexidade tecnológica das empresas parceiras.

Em síntese, as análises realizadas nesse trabalho contribuíram para o estudo das interações universidade-sociedade no Brasil. Demonstrou-se que os grupos de pesquisa no Rio Grande do Sul apresentam interações com diversos agentes da sociedade, para além das instituições que visam ao lucro. Esse engajamento social da universidade não pode ser ignorado, visto que possui potencial de contribuir para o bem-estar da sociedade.

\section{Social Engagement in Universities: an analysis of university-society interactions in Rio Grande do Sul}

Abstract: Based on the recent discussion on the social engagement of the university, this article aims to understand how university-society interactions take place in Rio Grande do Sul. For this purpose, the 2016 Census from the Directory of Research Groups of the National Council for Scientific and Technological Development (DGP/CNPq) is used as database. The analyzes aims to identify which agents the southern universities interact with and what type of interactions occur, as well as the differences between the areas of knowledge and between public and private universities. It was identified that research groups from universities in Rio Grande do Sul interact with several agents, such as the government, associations, cooperatives, foundations, hospitals, firms and other universities, located mainly in the south and southeast of Brazil. 
Keywords: University-society interactions; National Innovation Systems; Research Groups.

\section{Referências bibliográficas}

AROCENA, R; GÖRANSSON, B; SUTZ, J. Develpmental Universities in Inclusive Innovation systems. Suiça: Cham, 2018. E-book. Disponível em: https://link.springer.com/book/10.1007\%2F9783-319-64152-2. Acesso em: 24 jan. 2021.

DIRETÓRIO DOS GRUPOS DE PESQUISA NO BRASIL. Censo de 2016 do DGP/CNPQ. Brasília: CNPQ. Disponível em: http://dgp.cnpq.br/dgp/. Acesso em: 3 nov. 2020.

FREEMAN, C. Technology policy and economic performance: lessons from Japan. London: Pinter Pub, 1987.

GODDARD, J. Re-inventing the civic university: National Endowment for Science, Technology and the Arts. Inglaterra: NESTA provocation 12, 2009.

KRUSS, G. et al. Academic Interaction with social partners. Capetown: Human Sciences Research Council Press, 2012.

LUNDVALL, B. National Innovation Systems: Towards a Theory of Innovation and Interactive Learning. London: Pinter Publishers, 1992.

LUNDVALL, B. Higher education, innovation and economic development. In: WORLD BANK'S REGIONAL BANK CONFERENCE ON DEVELOPMENT ECONOMICS, 10, 2007, Beijing. Anais [...], Beijing, 2007.

MARCELLINO, I. S. et al. University-society collaboration in developing countries: Preliminary evidences from Brazil. In: ENCONTRO NACIONAL DE ECONOMIA INDUSTRIAL E INOVAÇÃO, 4., 2019, Campinas. Anais [...]. Campinas: ENEII, 2019.

MORA, J. et al. O engajamento social como motor do desenvolvimento regional: a contribuição das universidades latino-americanas. In: SERRA, M. et al. (org). Universidades e Desenvolvimento Regional: as Bases para a Inovação Competitiva. Rio de Janeiro: Ideia D, 2018.

NELSON, R. National Innovation Systems: A Comparative Analysis. New York: Oxford University Press, 1993.

SUZIGAN, W. ALBUQUERQUE, E. M. The Underestimated Role of Universities for the Brazilian System of Innovation. Brazilian Journal of Political Economy, São Paulo, v. 31, n. 1, p. 3-30, 2011. 